\title{
Self-Diagnosis of Surgical Site Infections: Lessons from a tertiary care centre in Karachi, Pakistan
}

\author{
Sana Z Sajun ${ }^{1}$, Katherine Albutt², Umme Salama Moosajee \\ Gustaf Drevin ${ }^{4}$, Swagoto Mukhopadhyay ${ }^{5}$, Lubna Samad ${ }^{6}$
}

\begin{abstract}
Background and Objective: Surgical site infections (SSIs) usually manifest post-discharge, rendering accurate diagnosis and treatment challenging, thereby catalyzing the development of alternate strategies like self-monitored SSI surveillance. This study aimed to evaluate the diagnostic accuracy of patients and Infection Control Monitors (ICMs) to develop a replicable method of SSI-detection.

Methods: A two-year prospective diagnostic accuracy study was conducted in Karachi, Pakistan between 2015 and 2017. Patients were educated about SSIs and provided with questionnaires to elicit symptoms of SSI during post-discharge self-screening. Results of patient's self-screening and ICM evaluation at followups were compared to surgeon evaluation.

Results: A total of 348 patients completed the study, among whom 18 (5.5\%) developed a SSI. Patient selfscreening had a sensitivity of $39 \%$, specificity of $95 \%$, positive predictive value (PPV) of $28 \%$, and negative predictive value (NPV) of $97 \%$. ICM evaluation had a sensitivity of $82 \%$, specificity of $99 \%$, PPV of $82 \%$, and NPV of $99 \%$.

Conclusion: Patients cannot self-diagnose a SSI reliably. However, diagnostic accuracy of ICMs is significantly higher and they may serve as a proxy for surgeons, thereby reducing the burden on specialized surgical workforce in LMICs. Regardless, supplementing post-discharge follow-up with patient self-screening could increase SSI-detection and reduce burden on health systems.
\end{abstract}

KEYWORDS: Surgical Site Infection, Patient Self-screening, Low-and Middle-Income Countries, Pakistan.

doi: https://doi.org/10.12669/pjms.36.ICON-Suppl.1716

How to cite this:

Sajun SZ, Albutt K, Moosajee US, Drevin G, Mukhopadhyay S, Samad L. Self-Diagnosis of Surgical Site Infections: Lessons from a tertiary care centre in Karachi, Pakistan. Pak J Med Sci. Special Supplement ICON 2020. 2020;36(1):S55-S60. doi: https://doi.org/10.12669/pjms.36.ICON-Suppl.1716

This is an Open Access article distributed under the terms of the Creative Commons Attribution License (http://creativecommons.org/licenses/by/3.0), which permits unrestricted use, distribution, and reproduction in any medium, provided the original work is properly cited.

1. Sana Z Sajun, MSc

Interactive Research and Development, Karachi, Pakistan

2. Katherine Albutt, MD MPH.

Department of Surgery, Massachusetts General Hospital,

3. Umme Salama Moosajee, BSc.

4. Gustaf Drevin,

5. Swagoto Mukhopadhyay, MD.

Program in Global Surgery and Social Change,

Harvard University, Department of Surgery, Boston, USA.

6. Lubna Samad, MRCS FCPS.

2,4: Program in Global Surgery and Social Change, Harvard Medical School, Boston, USA.

3,6: Center for Essential Surgery and Acute Care, Global Health Directorate,

Indus Health Network, Karachi, Pakistan

Correspondence:

Lubna Samad, MRCS FCPS.

Postal Address: The Indus Hospital, Korangi Crossing,

Karachi 75190, Pakistan.

E-mail: lubna.samad@ird.global

* Received for Publication:

September 23, 2019

* Accepted for Publication:

November 17, 2019

\section{INTRODUCTION}

Surgical site infections (SSIs) are the most common hospital-acquired infection in low- and middleincome countries (LMICs), which are estimated to occur in one in every ten surgical patients ${ }^{1,2}$ contrary to an incidence rate of $2.6 \%$ and $2.9 \%$ in the USA and European countries respectively. ${ }^{1}$ In Pakistan, the best available evidence suggests that SSIs occur in between $4 \%$ and $12.5 \%$ of procedures. ${ }^{3,4}$ SSIs are a chief cause of morbidity and mortality that raise the cost of treatment for patients and tax already fragile health systems. ${ }^{5,6}$

Early detection and appropriate diagnosis is critical to reduce SSI-associated morbidity and mortality ${ }^{5-7}$ Approximately, $84 \%$ of SSIs occur after a patient is discharged from the hospital, creating 
a host of surveillance and diagnostic challenges. ${ }^{2,5}$ These challenges are compounded in LMICs as patients are often lost to follow-up or are unable to travel long distances to seek care. Limitations of conventional methods of SSI-detection, coupled with the increasing importance being given to SSI incidence as a quality of care metrics have catalyzed the development of alternate surveillance strategies.

Hence, patient's ability to self-detect SSIs post-discharge is increasingly being explored., 8,9 Evidence from high-income countries (HICs) validates patients' ability to diagnose wound complications with reasonable accuracy..$^{9}$ Patientbased surveillance therefore denotes a cost-effective mode of post-discharge SSI-detection that alleviates the overworked healthcare providers. However, limited evidence is available from LMICs on selfmonitored surveillance of SSIs. ${ }^{1}$

A patient-centered self-screening tool was developed to assess patients' ability to accurately detect SSIs. The accuracy of patient self-screening and evaluation by Infection Control Monitors (ICMs) was analyzed to develop a simple, accurate, and reproducible method of SSI-detection.

\section{METHODS}

A 24-month prospective diagnostic accuracy study was designed and implemented to evaluate the SSI self- and ICM-screening questionnaire between October 2015 and September 2017. The study was granted ethics approval by the Institutional Review Boards at The Indus Hospital (Interactive Research and Development IRB: IRD_IRB_2015_09_003) and the Boston Children's Hospital- (IRB-P00020515).
The study was conducted at The Indus Hospital (TIH) - a 375-bed private tertiary care facility offering free-of-cost healthcare in the densely populated city of Karachi, Pakistan. TIH performs 6,000 operations annually for a nationwide catchment population, covering a variety of services. Surgical patients at TIH are scheduled for routine follow-up appointments at 14 and 30 postoperative days, during which a surgeon assesses overall recovery and identifies any post-operative complications.

All consecutive patients undergoing any of the 14 preselected surgical procedures (Table-I) at TIH were approached, consented and enrolled into the study. A priori sample size of 317 patients was calculated, using a web-based calculator, ${ }^{10}$ based upon an alpha of 0.01 , power of $90 \%$, assumed proportion of no event-event (no SSI by surgeon and SSI by patient or ICM) of $11 \%$, proportion of event-no event (SSI by surgeon and no-SSI by patient or ICM) of $1.83 \%$ from the pilot study and an attrition rate of $30 \%$.

An SSI was diagnosed and classified based upon the Center for Disease Control and Prevention (CDC) defined criteria i.e. "potential complications associated with a surgical procedure that typically occur at the surgical incision site within 30 post-operative days". ${ }^{11}$ SSIs were diagnosed by a surgeon if at least one of the symptoms was present (Fig.1). Surgeon diagnosis of SSIs was considered as gold standard for comparison with other diagnoses. All surgeries were classified using CDC criteria as being clean, clean-contaminated, contaminated and dirty, depending on the extent of microbial

\begin{tabular}{|c|c|c|c|c|c|}
\hline & Procedure & $\begin{array}{l}\text { Total Patient } \\
\text { Volume n }(\%)\end{array}$ & $\begin{array}{c}\text { SSI }(n=18) \\
n(\%)\end{array}$ & $\begin{array}{c}\text { No SSI }(n=330) \\
n(\%)\end{array}$ & $\begin{array}{c}\text { Chi-square } \\
\text { p-value }\end{array}$ \\
\hline \multirow[t]{3}{*}{ Orthopedics } & ORIF of Fracture & 66 (18.9) & $4(22.2)$ & $62(18.8)$ & 0.72 \\
\hline & Dynamic Hip Screw Arthoplasty & $9(2.6)$ & - & $9(2.7)$ & 0.48 \\
\hline & Joint Replacement & $3(0.9)$ & - & $3(0.9)$ & 0.68 \\
\hline General Surgery & Herniorraphy & $70(20.1)$ & $4(22.2)$ & $66(20.0)$ & 0.82 \\
\hline Cardiothoracic & Coronary Artery Bypass Graft & $33(9.5)$ & $3(16.7)$ & $30(9.1)$ & 0.29 \\
\hline \multirow[t]{2}{*}{ Urology } & Orchidopexy & $3(0.9)$ & - & $3(0.9)$ & 0.68 \\
\hline & Cystolithotomy & $1(0.3)$ & - & $1(0.3)$ & 0.82 \\
\hline \multirow[t]{5}{*}{ Pediatric Surgery } & ORIF of Fracture & $14(4.0)$ & $2(11.1)$ & $12(3.6)$ & 0.12 \\
\hline & Herniorraphy & $58(16.7)$ & $1(5.6)$ & $57(17.3)$ & 0.19 \\
\hline & Hydrocele Excision & $1(0.3)$ & - & $1(0.3)$ & 0.82 \\
\hline & Cystolithotomy & $1(0.3)$ & - & $1(0.3)$ & 0.82 \\
\hline & Orchidopexy & $5(1.4)$ & - & $5(1.5)$ & 0.60 \\
\hline E.N.T & Thyroid Surgery & $2(0.6)$ & - & $2(0.6)$ & 0.74 \\
\hline $\mathrm{Ob} / \mathrm{Gyn}$ & Cesarean Section & $82(23.6)$ & $4(22.2)$ & 78 (23.6) & 0.89 \\
\hline
\end{tabular}


contamination. ${ }^{12}$ Additionally, patients were categorized according to the WAMI Index, a proxy for socioeconomic status in LMICs. ${ }^{13}$

Moreover, an easy-to-use self-administered questionnaire was designed to elicit signs and symptoms of SSI through yes-or-no questions. This questionnaire was translated into Urdu, pretested on a small sample of patients and guardians and revised according to the feedback received. All enrolled patients received the questionnaire, a pictorial educational brochure on SSIs, and wound management counseling by one of the four designated ICMs, holding a degree in Bachelors of Nursing, post-surgery. Patients were advised to assess their surgical wounds at regular intervals post-discharge and were asked to fill the selfscreening questionnaire at every screening. The signs and symptoms elicited from the questionnaire are detailed in Fig.1. Patients were advised to call the helpline immediately if they experienced any symptoms of SSI. Patients who called in with at least one positive symptom were scheduled for an appointment with the relevant surgical team within 24-48 hours. Rest of the patients attended follow-up appointments as planned.

Data were analyzed using SPSS version 21.0. Descriptive statistics were computed for each study variable. The sensitivity, specificity, positive predictive value (PPV), and negative predictive values (NPV) of patients' and ICMs' ability to detect SSIs compared with surgeons' were calculated. Bivariate analysis was used to measure the association between any two variables of interest. Gwet's AC1 was calculated to estimate the degree of inter-rater reliability in diagnosing a SSI. ${ }^{14}$ Additionally, logistic regression was performed to assess for a correlation between a patient's ability to correctly self-screen for a SSI and their socio-economic status.

\section{RESULTS}

Demographics: A total of 454 patients consented and were enrolled into the study. Among these, 88 patients were lost to follow-up and 18 patients were excluded due to postponed surgical procedures or death unrelated to SSI, leaving a total of 348 who completed the study. Basic demographics of participants are presented (Table-II). A breakdown of study procedures is presented (Table-I). All surgeries were classified as "clean".

SSI Diagnosis: A total of 18 patients were diagnosed with SSIs, equating to a prevalence of $5.2 \%$. Patients who developed an SSI did not differ from the rest in terms of demographic characteristics. There was no relationship between socioeconomic status, as measured by participants' WAMI score, and presence or absence of SSI $(p=0.22)$. There was no relationship between SSI diagnosis and surgical procedures.

Self-Screening: Patients correctly reported an SSI in seven $(38.9 \%)$ cases and correctly noted the absence of an SSI in $312(94.5 \%)$ cases. In 11 $(61.1 \%)$ cases, an infection was diagnosed by the surgeon during a routine post-operative visit but was not reported by the patient. In 18 (5.5\%) cases, patients suspected a SSI but the surgeon did not

\begin{tabular}{|c|c|c|c|c|c|c|c|c|c|c|c|c|c|c|c|c|c|c|}
\hline Diagnosis & \multicolumn{6}{|c|}{ Patient, Surgeon \& ICM } & $\begin{array}{l}\text { Pat } \\
\&\end{array}$ & \multicolumn{10}{|c|}{ Surgeon \& ICM } & \multirow{2}{*}{$\begin{array}{c}\text { Surg } \\
18 \\
\end{array}$} \\
\hline SSI Cases & 1 & 2 & 3 & 4 & 5 & 6 & 7 & 8 & 9 & 10 & 11 & 12 & 13 & 14 & 15 & 16 & 17 & \\
\hline Drainage & " & * & 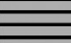 & \% & E & 世" & $\bar{\nu}$ & * & & * & * & & & $*$ & * & & * & \\
\hline Pain & & & * & 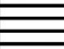 & 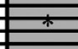 & 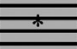 & 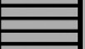 & * & * & * & * & & & & * & * & & \\
\hline Redness & & & & $\overline{\overline{\bar{~}}}$ & है & 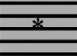 & $\bar{~}$ & & & * & * & & & * & & & & \\
\hline Fever & & en & " & 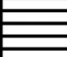 & 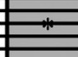 & & 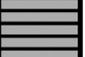 & & * & & & & * & & & * & & \\
\hline Swelling & * & * & $\bar{E}$ & 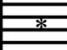 & F & 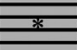 & & & * & * & * & & * & * & * & * & & \\
\hline $\begin{array}{c}\text { Edge } \\
\text { separation }\end{array}$ & * & & * & & & $\overline{\bar{Z}}$ & 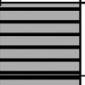 & & & * & & * & * & & * & & & \\
\hline Warmth & एक & & & & 类 & $*$ & & & & & & & & * & * & & & \\
\hline $\begin{array}{ll} & \text { Patie } \\
& \text { Pat: } 1\end{array}$ & $\begin{array}{l}\text { t- iden } \\
\text { atient }\end{array}$ & ied $s$ & iptom & & $\begin{array}{l}\text { ICM ic } \\
\text { CM: Inf }\end{array}$ & $\begin{array}{l}\text { entified } \\
\text { ction } C\end{array}$ & ontrol I & Ionitor & & & $\begin{array}{l}\text { Surge } \\
\text { Surg: }\end{array}$ & $\begin{array}{l}\text { iden } \\
\text { argeo }\end{array}$ & ied $s$. & ptom & & & & \\
\hline
\end{tabular}

Fig.1: CDC Defined Symptoms Identification Matrix for true positive SSIs. 
Sana Z Sajun et al.

Table-II: Patient demographics.

\begin{tabular}{|c|c|c|c|c|c|}
\hline & & Total n $(\%)$ & $S S I(n=18) n(\%)$ & No SSI $(n=338) n(\%)$ & Chi-square p-value \\
\hline Age & Mean \pm SD & $30.8 \pm 19.4$ & $37.8 \pm 18.8$ & $30.4 \pm 19.4$ & $0.12^{*}$ \\
\hline \multirow{2}{*}{ Gender } & Female & $139(39.9)$ & 8 (44.4) & 131 (39.7) & 0.81 \\
\hline & Male & $209(60.1)$ & $10(55.6)$ & $199(60.3)$ & \\
\hline \multirow[t]{2}{*}{ Marital Status } & Single & 116 (33.3) & $3(16.7)$ & $113(34.2)$ & 0.20 \\
\hline & Married & $232(66.7)$ & 15 (83.3) & $217(65.8)$ & \\
\hline \multirow[t]{2}{*}{ Area } & Urban & $328(94.3)$ & $16(89.9)$ & 312 (94.5) & 0.28 \\
\hline & Rural & $20(5.7)$ & 2 (11.1) & $18(5.5)$ & \\
\hline Patient's & Urdu & $243(69.8)$ & $10(55.6)$ & 233 (70.6) & 0.20 \\
\hline Primary & Sindhi & $27(7.8)$ & $1(5.6)$ & $26(7.9)$ & \\
\hline \multirow[t]{3}{*}{ Language } & Punjabi & $22(6.3)$ & $2(11.1)$ & $20(6.1)$ & \\
\hline & Pushto & $24(6.9)$ & $1(5.6)$ & $23(7.0)$ & \\
\hline & Other & $32(9.2)$ & $4(22.2)$ & $28(8.5)$ & \\
\hline Formal & Yes & $224(64.4)$ & $12(66.7)$ & $212(64.2)$ & 1.00 \\
\hline Education & No & 124 (35.6) & $6(33.3)$ & $118(35.8)$ & \\
\hline Education Years† & Mean \pm SD & $4.8 \pm 1.4$ & $4.7 \pm 1.1$ & $4.8 \pm 1.4$ & $0.77^{*}$ \\
\hline WAMI Score $\ddagger$ & Mean \pm SD & $0.72 \pm 0.1$ & $0.75 \pm 0.1$ & $0.72 \pm 0.1$ & $0.22^{*}$ \\
\hline
\end{tabular}

†Education Status of Caregiver/Parent was recorded for patients unable to read or write/pediatric patients. $\ddagger$ Water and sanitation, Assets, Maternal education, and monthly household Income (WAMI) Index;

Ranges between 0-1. * t-test was used to calculate p-vales for Age, Education Years and WAMI Score.

confirm these findings (Table-III). The sensitivity of the screening tool was $38.9 \%$ (95\% CI [18.3, 63.9]), specificity was $94.5 \%$ (95\% CI [91.4, 96.6]), PPV was $28.0 \%(95 \%$ CI $[12.9,49.6])$ and NPV was 96.6\% (95\% CI [93.8, 98.2]).

ICM Diagnosis: ICMs correctly reported a SSI in $14(82.4 \%)$ cases and correctly noted the absence of a SSI in $327(99.1 \%)$ cases. There were three false negative cases and three false positive cases (Table-III). The sensitivity of ICM evaluation was $82.4 \%$ (95\% CI [55.8, 95.3]), specificity was $99.1 \%$ (95\% CI [97.1, 99.8]), PPV was 82.4\% (95\% CI [55.8, 95.3]) and NPV was 99.1\% (95\% CI [97.1, 99.8]).

SSI Symptoms: The most common signs/symptom used for SSI identification amongst patients was drainage $(n=6,85.7 \%)$ followed by pain at the incision site $(n=4,57.1 \%)$, redness $(n=4,57.1 \%)$, fever $(n=4,57.1 \%)$ and swelling $(n=4,57.1 \%)$. The most commonly documented symptoms amongst ICMs were swelling $(n=11,78.6 \%)$, drainage $(n=10$, $71.4 \%)$, pain $(\mathrm{n}=8,57.1 \%)$, and wound separation $(n=7,50.0 \%)$. Surgeons detected drainage $(n=13$,
$72.2 \%)$ most commonly, followed by pain $(n=11$, $61.1 \%)$, wound separation $(\mathrm{n}=11,61.1 \%)$, redness $(n=10,55.6 \%)$, and swelling $(n=10,55.6 \%)$. There was no statistically significant difference between the symptoms used to identify an SSI and the type of identifier $(p=0.99)$. Clustering of reported symptoms by patients, ICMs and surgeons, respectively is presented in a matrix (Fig.1).

\section{DISCUSSION}

The SSI prevalence in this study was $5.2 \%$, which is consistent with the existing estimates of SSI incidence in Pakistan i.e. between $4 \%$ and $12.5 \%{ }^{3,4}$ The relatively low rate of SSI could be attributed to all included surgeries being classified as "clean". ${ }^{2-4}$ This rate is comparable to $4 \%$ SSI prevalence for "clean" cases in Zafar et al.'s multicenter study. ${ }^{3}$ It is important to note, regardless of low SSI prevalence, that SSI-associated morbidity and mortality are preventable making early detection and appropriate diagnosis essential to improving outcomes..$^{15}$

Table-III: Patient and ICM Diagnoses vs. Surgeon Diagnosis.

\begin{tabular}{|c|c|c|c|c|c|c|c|}
\hline & & \multicolumn{2}{|c|}{ Patient self-screening $n(\%)$} & \multirow[t]{2}{*}{ Total } & \multicolumn{2}{|c|}{ ICM evaluation $n(\%)$} & \multirow[t]{2}{*}{ Total } \\
\hline & & $S S I$ & No SSI & & SSI & No SSI & \\
\hline \multirow{2}{*}{$\begin{array}{l}\text { Surgeon Diagnosis } \\
(\mathrm{n} \%)\end{array}$} & SSI & $7(38.9)$ & $11(61.1)$ & $18(100.0)$ & $14(82.4)$ & $3(17.6)$ & $17(100.0)$ \\
\hline & No SSI & $18(5.5)$ & $312(94.5)$ & $330(100.0)$ & $3(0.9)$ & $327(99.1)$ & $330(100.0)$ \\
\hline Total & & $25(7.2)$ & $323(92.8)$ & $348(100.0)$ & $17(4.9)$ & $330(95.1)$ & $347(100.0) \dagger$ \\
\hline
\end{tabular}

$\dagger \mathrm{ICM}$ results were calculated from a total of 347 observations; 1 case was omitted as the patient presented in the emergency

room at The Indus Hospital out of study hours; as a result, a study ICM was not present at the time for evaluation. 
In this study, patients were unable to accurately self-diagnose SSIs. With a self-screening sensitivity of only $38.9 \%$, patients failed to diagnose SSIs in over half of the confirmed SSIs. Existing literature is highly variable in terms of the accuracy of patient self-screening. ${ }^{8,16}$ Studies that compared patient self-diagnosis with health professional diagnosis found substantial agreement between the two parties' independent wound assessment with a few discordant assessments.

The variable results of self-screening have motivated some practitioners to explore techniques like telephone surveillance. Richter et al. found a sensitivity of only $66 \%$ in a meta-analysis of telephonic SSI surveillance. ${ }^{16}$ Others have suggested the supplementation of self-screening with photo documentation. For example, mPOWER uses Smartphone technology in the US to allow patients to share surgical wound photos and answer SSI triage questions during the post-discharge period. ${ }^{17}$ In LMICs, mobile and network requirements for effective Smartphone photo follow-up are a limiting factor in bringing such technology to scale, which may change in the coming years. A near perfect agreement was found between surgeon and ICM assessments at follow-up with high sensitivity and specificity, suggesting that ICMs can correctly diagnose SSIs. Other studies corroborate this finding suggesting that trained non-physicians can correctly identify SSIs and may be used as a proxy to surgeons for SSI-detection. ${ }^{18,19}$ In LMICs, taskshifting and task-sharing have been promoted as a response to the healthcare workforce crisis. ${ }^{20}$

Drainage was found to be the most frequent symptom identified by all cadres in true positive cases, which reinforces Whitby et al's observation of discharge being the most common symptom used for SSI-detection by patients. However, drainage was often confused with serous discharge as opposed to infectious pus resulting in an overestimation of infection rates by the study patients. ${ }^{8}$ In our study, drainage was also identified in half of the false positive cases suggesting that the presence of drainage is not highly specific for SSIs. Despite extensive education regarding worrisome drainage characteristics, patients were unable to correctly identify pathologic wound drainage, resulting in over-identification of this symptom. Future patient-centered SSI interventions should emphasize the spectrum of discharge. Further research into constellation of signs and symptoms that best correlate with correct SSI diagnosis is necessary.
Limitations of the study: First, the power to detect significant differences among groups was limited among this cohort owing to the low SSI prevalence rate. Second, the study failed to capture the true health-seeking pattern of patients by disregarding whether the patients self-screened at regular intervals using the questionnaire or if they self-screened but did not call in. Future interventions should include reminder systems to prompt patients to self-screen at regular intervals and encourage patients to come in for follow-up irrespective of SSI suspicion. Third, a $20 \%$ loss to follow-up rate made it impractical to consider ICM screening as the only form of SSI surveillance. Therefore, further research involving community health workers would help address this concern and provide an alternative to facility-based screening.

\section{CONCLUSION}

This study employed a patient-centered tool for active post-discharge surveillance of SSIs in Pakistan. While patients were unable to accurately diagnose SSIs, ICMs may be used as a proxy to surgeons for SSI-detection, thereby reducing the burden on the specialized surgical workforce in LMICs. Supplementing regular post-discharge follow-up with ICM-screening in low-resource settings has the potential to increase the rate of SSI- detection with minimal additional burden to the health system.

Acknowledgements: We are grateful to the patients and providers that participated. We would like to thank Dr. John Meara for his kind support, valuable feedback and guidance throughout the study duration and manuscript's compilation. We are also thankful to Ms. Alizeh Ahmad for overseeing research activities and helping with data collection. We would also like to thank Dr Naila Baig Ansari and Sundus Iftikhar at TIH Research Center for their statistical support throughout the study as well as Dr Altaf Ahmed and the Infection Control Department at TIH for training the ICMs and their unwavering support.

\section{Conflict Interests: None.}

Funding: Collaborative Research AwardHarvard Medical School (HMS), Centre of Global Health Delivery- Dubai, UAE; Ronda Stryker and William Johnston Global Surgery Fellowship Fund, and Program in Global Surgery and Social Change at HMS. 


\section{REFERENCES}

1. Allegranzi B, Bagheri Nejad S, Combescure C, Graafmans W, Attar H, Donaldson L, et al. Burden of endemic health-careassociated infection in developing countries: Systematic review and meta-analysis. Lancet. 2011;377(9761):228-241. doi: 10.1016/S0140-6736(10)61458-4.

2. Bhangu A, Ademuyiwa AO, Aguilera ML, Alexander P, Al-Saqqa SW, Borda-Luque G, et al. Surgical site infection after gastrointestinal surgery in high-income, middle-income, and low-income countries: a prospective, international, multicentre cohort study. Lancet Infect Dis. 2018;18(5):516-525.

3. Malik AZ. Surgical site infections after elective surgery in Pakistan: Surgipak Study. J Rawalpindi Med Coll. 2015;19(3):209-214.

4. Ling ML, Apisarnthanarak A, Madriaga G. The Burden of Healthcare-Associated Infections in Southeast Asia: A Systematic Literature Review and Meta-analysis. Clin Infect Dis. 2015;60(11):1690-1699. doi: 10.1093/cid/civ095

5. Oliveira AC, Lima ED, Lima Ade P. Post-discharge surgical site infection surveillance in a hospital in Brazil. Am J Surg. 2007;193(2):266-268. doi: 10.1016/j.amjsurg.2006.05.016

6. Fehr J, Hatz C, Soka I, Kibatala P, Urassa H, Smith T, et al Risk factors for surgical site infection in a Tanzanian district hospital: a challenge for the traditional National Nosocomial Infections Surveillance system index. Infect Control Hosp Epidemiol. 2006;27(12):1401-1404. doi: 10.1086/509855

7. Humphreys H. Preventing surgical site infection. Where now? J Hosp Infect. 2009;73(4):316-322. doi: 10.1016/j. jhin.2009.03.028

8. Whitby M, McLaws M, Collopy B, Looke D, Doidge S, Henderson B, et al. Post-discharge surveillance: can patients reliably diagnose surgical wound infections? J Hosp Infect. 2002;52(3):155-160.

9. Wilson AP, Hodgson B, Liu M, Plummer D, Taylor I, Roberts $\mathrm{J}$, et al. Reduction in wound infection rates by wound surveillance with postdischarge follow-up and feedback. Br J Surg. 2006;93(5):630-638. doi: 10.1002/bjs.5303

10. Sample Size Calculator: HyLown.com. Available from: http://powerandsamplesize.com/Calculators/ComparePaired-Proportions/McNemar-Z-test-2-Sided-Equality.

11. Horan TC, Gaynes RP, Martone WJ, Jarvis WR, Emori TG. CDC definitions of nosocomial surgical site infections, 1992: a modification of CDC definitions of surgical wound infections. Infect Control Hosp Epidemiol. 1992;13(10):606-608
12. Wolff SP, Garner A, Dean RT. Free radicals, lipids and protein degradation. Trends Biochem Sci. 1986;11(1):27-31.

13. Psaki SR, Seidman JC, Miller M, Gottlieb M, Bhutta $\mathrm{ZA}$, Ahmed $\mathrm{T}$, et al. Measuring socioeconomic status in multicountry studies: results from the eight-country MAL-ED study. Popul Health Metr. 2014;12(1):8. doi: 10.1186/1478-7954-12-8

14. Gwet K. The definitive guide to measuring the extent of agreement among raters: Hand book of inter-rater reliability fourth edition. Advanced Analytics LLC; 2014.

15. Control CfD, Prevention. National Healthcare Safety Network Patient Safety Component Manual [Internet]. Atlanta, GA: CDC. 2014.

16. Richter V, Cohen MJ, Benenson S, Almogy G, Brezis M. Patient self-assessment of surgical site infection is inaccurate. World J Surg. 2017;41(8):1935-1942.

17. Evans HL, Lober WB. A pilot use of patient-generated wound data to improve postdischarge surgical site infection monitoring. JAMA Surg. 2017;152(6):595-596.

18. Weigelt JA, Dryer D, Haley RW. The necessity and efficiency of wound surveillance after discharge. Arch Surg. 1992;127(1):77-81; discussion 81-72. doi: 10.1001/ archsurg.1992.01420010091013

19. Mangram A. Guideline for prevention of surgical site infection, 1999. Hospital Infection Control Practice Advisory Committee. Infect Control Hosp Epidemiol. 1999;20:250-278.

20. Matousek A, Paik K, Winkler E, Denike J, Addington SR, Exe $\mathrm{C}$, et al. Community health workers and smartphones for the detection of surgical site infections in rural Haiti: a pilot study. Lancet. 2015;385(Suppl 2):S47. doi: 10.1016/ S0140-6736(15)60842-X

\section{Authors' Contributions:}

SZS \&SM:Conceptualized, designed, implemented, analyzed data and did manuscript writing, takes responsibility for integrity of research.

KA \& GD: Analyzed data and drafted the final manuscript.

USM: Helped implement the research, analyze data and draft the final manuscript.

LS: Helped conceptualize, design and monitor the research and proofread the manuscript. 